\title{
An invariance property of diffusive random walks
}

\author{
BLANCO StePhANE ${ }^{1}$ and FOURNIER RICHARD ${ }^{1}$ \\ 1 Laboratoire d'Energétique - Université Paul Sabatier, 118 route de Narbonne, 31062 \\ Toulouse Cedex 4
}

PACS. 05.60.Cd - Classical transport.

PACS. 05.40.-a - Fluctuation phenomena, random processes, noise, and Brownian motion.

\begin{abstract}
Starting from a simple animal-biology example, a general, somewhat counterintuitive property of diffusion random walks is presented. It is shown that for any (nonhomogeneous) purely diffusing system, under any isotropic uniform incidence, the average length of trajectories through the system (the average length of the random walk trajectories from entry point to first exit point) is independent of the characteristics of the diffusion process and therefore depends only on the geometry of the system. This exact invariance property may be seen as a generalization to diffusion of the well known mean-chord-length property 1], leading to broad physics and biology applications.
\end{abstract}

Let us first consider a practical animal-biology example that was at the origin of the theoretical work reported hereafter. It is well established, for diverse species, that the spontaneous displacement of insects such as ants, on an horizontal planar surface, may be accurately modeled as a constant-speed diffusion random-walk 2 4 . We assume that a circle of radius $R$ is drawn on the surface and we measure the average time that ants spend inside the circle when they enter it (see Fig. 1). The last assumption is that measurements are performed a long time after ants were dropped on the surface, so that the memory of their initial position is lost : this is enough to ensure that no specific direction is favored and therefore that when ants encounter the circle, their incident directions are distributed isotropically. Simple Monte Carlo simulations of such experiments (see Fig. 2) indicate without any doubt that the average encounter time (time between entry into the circle and first exit), for a fixed velocity, depends only of the circle radius. It is independent of the characteristics of the diffusion walk : the mean free path $\lambda$ (average distance between two scattering events, i.e. between two direction-changes), and the single-scattering phase function $p\left(\mathbf{u}_{\mathbf{s}}, \mathbf{u}_{\mathbf{i}}\right)$ (probability density function of the scattering direction $\mathbf{u}_{\mathbf{s}}$ for an incident direction $\mathbf{u}_{\mathbf{i}}$ ). Furthermore, this average time scales as $R / v$, which means that the average trajectory-length $\langle L\rangle$ scales as $R$. The average trajectory-length would therefore be the same for different experiments with any insect species - or for any type of diffusive corpuscular motion.

There are two reasons why this observation may initially sound counter-intuitive. The first reason is that the average length of diffusion trajectories between two points is known to scale approximately as $d^{2} / \lambda$ where $d$ is the distance between the two points $\left({ }^{1}\right)$. For shorter mean

(1) This scaling would be exact for an infinite domain in the limit $d>>\lambda 5$

(c) EDP Sciences 
free paths, because of more frequent direction-changes, it should be required that ants cover more distance to go from one point to another (see Fig. 1). One could therefore expect an increase of $\langle L\rangle$ when reducing the mean free path.

The second reason is the following. When the mean free path is very small compared to $R$, most ants have an exit point very close to their entry point. This means that for analysis of short trajectories, the circle curvature may be forgotten. Consequently, there is an homothety of trajectories corresponding to a mean free path $\lambda$ and say a mean free path $\lambda / 2$ (see Fig. 3). This should imply that $\langle L\rangle$ decreases when reducing $\lambda$, which is the opposite conclusion of that corresponding to the first way of reasoning.

Obviously these two images are both meaningful, but the corresponding processes are simultaneously under way and compensate each other exactly. The first image concerns long trajectories (large numbers of scattering events before exit) and the second one concerns short trajectories (exit after a few scattering events). Why this exact compensation occurs is not obvious, but a simple proof can be proposed on the basis of transport theory.

Property. - Let us first generalize the underlying property. Consider any finite volume $\Omega$ of envelope $\Sigma$ submitted to a uniform isotropic particle-incidence. $\Omega$ is filled with any non-homogeneous anisotropic perfectly diffusing medium. For a statistical event in which a particle enters $\Omega$ through $\Sigma$, the total trajectory length $L$ is defined as the length of the multiple scattering trajectory from entry point to first exit through $\Sigma$. Then the average value of $L$ is independent of both the mean free path and single-scattering phase function fields $\left({ }^{2}\right)$ :

$$
<L>4 \frac{\Omega}{\Sigma}
$$

Note that this relationship may be seen as a generalization of the well known mean-chordlength property [1] that corresponds to Eq. 1 in the limit of an infinite mean free path (straight-line trajectories) $\left({ }^{3}\right)$.

Proof : The property addressed is purely geometric. In particular, if two sets of particles are considered, with same mean free path and same single-scattering phase function, $\langle L>$ will be identical, independently of the particle speed. This means that if it can be shown that the property is true for constant-speed diffusion, then the property is true for any particlediffusion process. Consequently, in order to establish the validity of Eq. 1, it is rigorously sufficient to derive a proof in the restricted case of a constant-speed displacement.

On this basis, the starting point is the following : one way of creating a uniform isotropic particle incidence is to consider that $\Omega$ is part of a larger diffusing system in a state of statistical equilibrium. The specific intensity $f(\mathbf{x}, \mathbf{u})$ (number of particles passing at a location $\mathbf{x}$, in a direction $\mathbf{u}$, per unit time, per unit normal area and per unit solid angle) is therefore uniform and isotropic : $f(\mathbf{x}, \mathbf{u})=f_{0}$. From this equilibrium state, let us imagine that a uniform particle-absorption, of absorption coefficient $\mu_{a}$ (inverse of the absorption mean-free-path), is added to the physics of $\Omega$. This means that the number of absorbed particles at a each location $\mathbf{x}$, per unit time and per unit volume, is $\mu_{a} v \int_{4 \pi} f(\mathbf{x}, \mathbf{u}) d \mathbf{u}$ where $v$ is the particle speed. This also means that for any particle traveling a distance $L$ through $\Omega$, the probability that an absorption occurs before exit is $1-\exp \left(-\mu_{a} L\right)$. In the stationary state, two ways of

\footnotetext{
$\left({ }^{2}\right)$ For two-dimension geometries Eq. 1 becomes $\langle L\rangle=\pi \frac{S}{P}$ where $S$ is the surface of the considered system and $P$ its perimeter.

$\left({ }^{3}\right)$ The assumption of isotropic incidence is formally equivalent to a cosine angular distribution. For instance, if $\Sigma$ is an infinite planear slab of thickness $e$, for straight-line trajectories $\left\langle L>\right.$ writes $\int_{0}^{\pi / 2} 2 \cos \theta \sin \theta l(\theta) d \theta$ with $l(\theta)=\frac{e}{\cos \theta}$, leading to $\langle L\rangle=2 e$, which is compatible with the mean-chord-length property with $\frac{\Omega}{\Sigma}=\frac{e}{2}$.
} 
expressing the total absorption rate inside $\Omega$ are therefore available : the first is to integrate the local absorption rate over the volume; the second is to consider the incident particle flux at the boundary $\pi v f_{0} \Sigma$ (the factor $\pi$ appears because of isotropy) and to integrate the absorptions over all possible trajectories trough $\Omega$. This leads to the following equation :

$$
\int_{\Omega}\left[\mu_{a} v \int_{4 \pi} f(\mathbf{x}, \mathbf{u}) d \mathbf{u}\right] d \mathbf{x}=\pi v f_{0} \Sigma \int_{0}^{\infty}\left[1-\exp \left(-\mu_{a} L\right)\right] p(L) d L
$$

where $p(L)$ is the probability density function of the trajectory length.

Let us now divide by $\mu_{a}$ both sides of Eq. 2 and take the limit $\mu_{a} \rightarrow 0$. At this limit, the system is again purely diffusing and statistical equilibrium is satisfied : $f(\mathbf{x}, \mathbf{u})=f_{0}$. The left hand side of the equation therefore becomes proportional to $\Omega$. On the right hand side, the limit $\frac{1}{\mu_{a}}\left[1-\exp \left(-\mu_{a} L\right)\right] \rightarrow L$ turns the integral into the average trajectory length $<L>=\int_{0}^{\infty} L p(L) d L$. Altogether we get :

$$
4 \pi v f_{0} \Omega=\pi v f_{0} \Sigma<L>
$$

which leads to Eq. 1.

Interest. - Such an invariance property $(<L>$ remaining constant when changing the random-walk characteristics) offers a challenging opportunity to test available physical pictures of particle diffusion. As illustrated in the introduction, only an exact compensation of physical trends associated with long and short trajectories can explain Eq. 1, but this compensation is hard to appreciate intuitively.

Outside the intrinsic theoretical interest of such a compensation effect, the property presented might allow a renewed look at the open question of modeling the statistics of shortlength diffusion trajectories. The statistics of long trajectories has been modeled successfully thanks to the assumption that the number of scattering events along each such trajectory is high, which allows the use of averaging relations [5]. As far as short trajectories are concerned, less successful work is reported, mainly because the corresponding statistical problem is of a much higher complexity 6 8]. Of course, Eq. 1 as such solves none of the corresponding problems, but the fact that constraint relationships can be exhibited between the statistics of short trajectories and long trajectories might be of direct interest for researchers in these fields.

As far as practical applications are concerned, despite the very wide range of physics and biology problems in which analysis of particle-diffusion trajectories are required, the following objection may be formulated : application of Eq. 1 requires that the considered system be purely diffusing and that the particle incidence be uniform and isotropic, both conditions that are sufficient to ensure that the particle distribution tends to statistical equilibrium, which turns the problem into one of limited interest. Present-day questions that involve detailed analysis of diffusion processes concern non-equilibrium conditions and Eq. 1 may therefore appear of little practical use.

One way of answering this objection is to refer to all configurations in which symmetry considerations allow to Eq. 1 to be applied under non-equilibrium conditions. Let us consider again the biology example used in the introduction. All entry points on the circle play a similar role, which in turn allows us to consider experiments in which only one entry location is offered. Such experiments are typical of conditions where the proposed property is applicable, although the distribution of ants inside the circle does not fulfill conditions for statistical equilibrium. Another more practical example along the same line may be seen in the field of radiative transfer for planetary atmospheres. The basic assumption is commonly that 
atmospheres may be divided into homogeneous plane-parallel horizontal layers. There are numerous conditions in which the assumption may be made that incident photons on each side are distributed quasi-isotropically. This is typically the case when photon sources are distributed within the atmosphere (as for infrared radiation in the earth's atmosphere, where collimated solar sources can be neglected [9] ). The upward and downward photon fluxes being distinct, each layer is not in a state of statistical equilibrium. However, for symmetry reasons, Eq. 1 may be applied separately to upward and downward photon incidences. Very similar illustrations could be found in neutron transport as well as in atomic charged-particle transport.

Another class of problems where Eq. 1 may be useful for analysis of non-equilibrium conditions is the following : problems where uniformity and isotropy of particle incidence is effectively satisfied (strictly or via symmetry assumptions), but where the system is not purely diffusing. For example, considering photon migration in turbid media, mainly for medicalimaging applications, it was shown that the photon propagation process could be separated into an absorption dependent part and a pure diffusion part 10,11. We used such a physical picture in the above presented proof, when writing the total absorption rate inside the system (in the case of a uniform absorption coefficient $\mu_{a}$ ) as $A=\pi v f_{0} \Sigma \int_{0}^{+\infty}\left[1-\exp \left(-\mu_{a} L\right)\right] p(L) d L$. In this expression, $L$ is indeed the trajectory length of photons across the system as if no absorption occurred and we can state that $\langle L\rangle=4 \frac{\Omega}{\Sigma}$. In the limit of a weak absorption for instance (optically thin absorption), the total absorption rate may therefore be approximated (linearizing the exponential) as

$$
A \approx \pi v f_{0} \Sigma \int_{0}^{+\infty} \mu_{a} L p(L) d L=\pi v f_{0} \Sigma \mu_{a}<L>=4 \pi \Omega v f_{0} \mu_{a}
$$

This property allows radiative transfer exchanges to be easily quantified inside systems of very complex geometries, as is already commonly performed in thermal-engineering in the limit of non-scattering radiation (thanks to the mean-chord-length property) 12. Equation 4 establishes that such engineering techniques may be rigorously extended to configurations with non-negligible photon scattering effects.

Finally, Eq. 1 may be experimentally used to test the relevance of diffusion assumptions, in particular in biology contexts where suspicions of external orientation or internal marking effects regularly arise. This is possible as soon as experimental techniques are implemented, allowing individual paths to be tracked and stored automatically, which is undeniably a common trend in present-day research into animal behavior. 


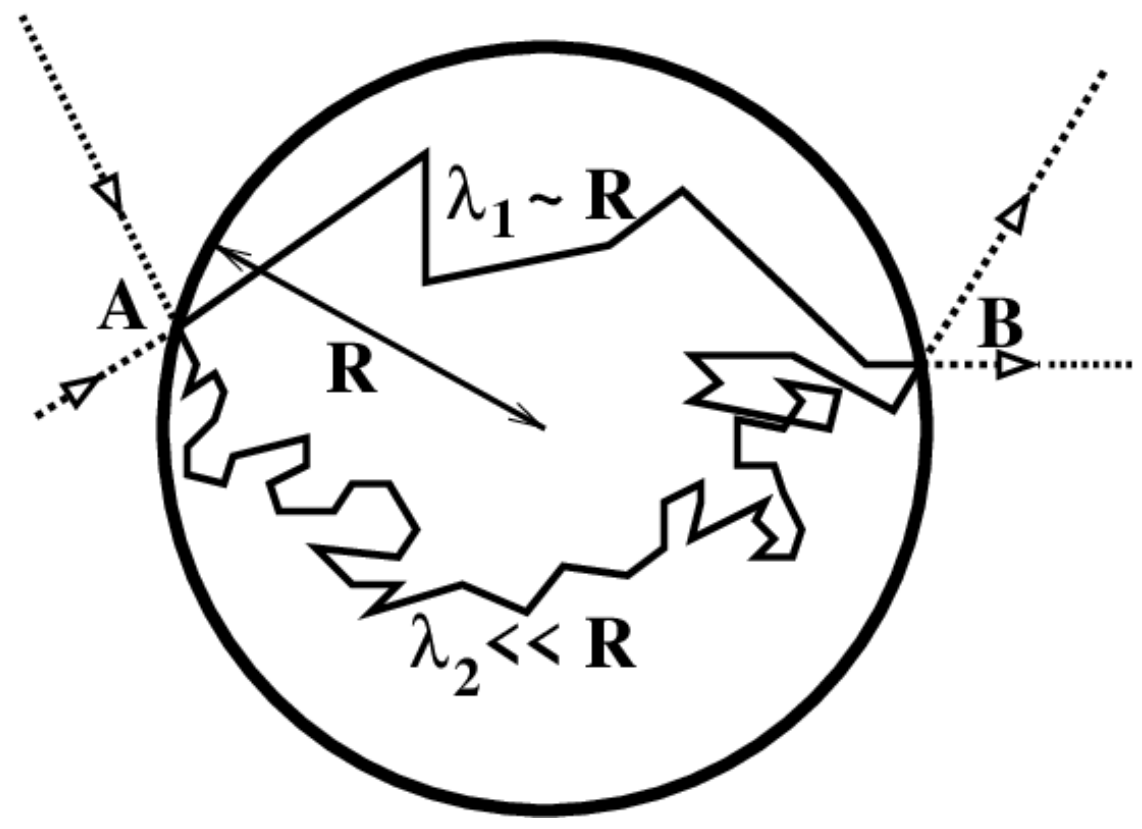

Fig. 1 - Random walk illustration - Trajectories of diffusing particles inside a circle of radius $R$, from entry point $A$ to exit point $B$. The first trajectory is typical of a particle with a mean free path $\lambda_{1}$ of the order of $R$, whereas the second trajectory corresponds to a mean free path $\lambda_{2}<\lambda_{1}$; this illustrates the fact that the trajectory lengths inside the circle, $L_{1}$ and $L_{2}$ respectively, should averagely verify $L_{1}<L_{2}$.

\section{REFERENCES}

[1] Case, K.M. and Zweifel, P.F., Linear Transport Theory (Addison-Wesley) 1967

[2] Turchin, P., Ecology, 72 (1991) 1253.

[3] Crist, T. O. and MacMaHon, J. A., Insectes Sociaux, 38 (1991) 379.

[4] Holmes, E. E., Am. Nat., 142 (1993) 6134.

[5] Morse, P. M. And Feshbach, H., Methods of Theoretical Physics (McGraw-Hil) 1953

[6] Freund, I. and Kaveh, M. and Rosenbluh, M., Phys. Rev. Lett., 60 (1988) 1130.

[7] Yoo, K. M. and Liu, F. and Alfano, R. R., Phys. Rev. Lett., 64 (1990) 2647.

[8] Ray, T. S. and Glasser, M. L. and Doering, C. R., Phys. Rev. A, 45 (1992) 8573.

[9] Goody, R. M. and Yung, Y. L., Atmospheric Radiation (Oxford University Press) 1989

[10] Perelman, L. T. and Wu, J. and Itzkan, I. and Feld, M. S., Phys. Rev. Lett., 72 (1994) 1341.

[11] Perelman, L. T. and Wu, J. and Wnag, Y. and Itzkan, I. and Dasari, R. R. and Feld, M. S., Phys. Rev. E, 51 (1995) 6134.

[12] Siegel, R. And Howell, J. R., Thermal Radiation Heat Transfer (McGraw-Hill) 1992 


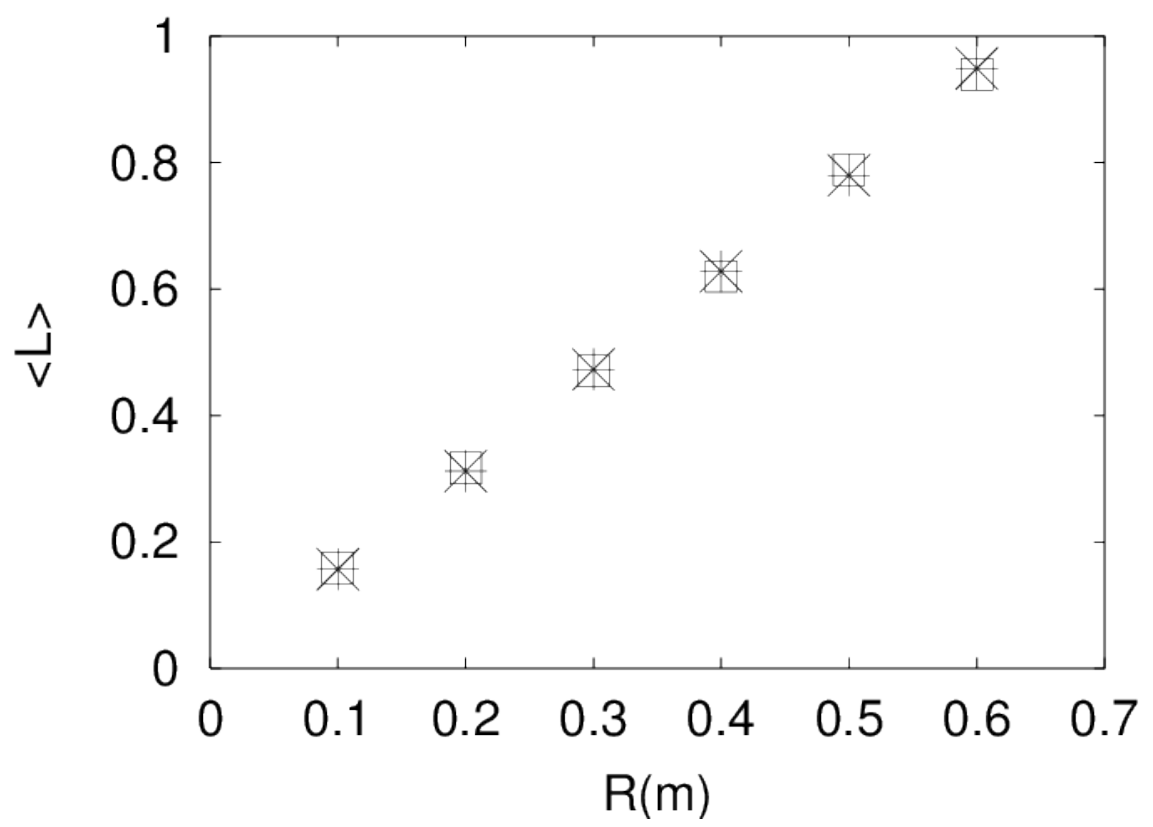

Fig. 2 - Monte Carlo simulations - Simulated average trajectory-length inside a circle of radius $R$ for two insects with distinct random-walk characteristics : $*$ mean-free-path $\lambda=5 \cdot 10^{-3} \mathrm{~m}$ (measured for the ant species Messor sancta L.); $\square$ mean-free-path $\lambda=5 \cdot 10^{-2} m$ (roughly estimated for the cockroach species Blattella germanica L.). In both simulations, the asymmetry parameter of the single scattering phase function is $g=0.5$.

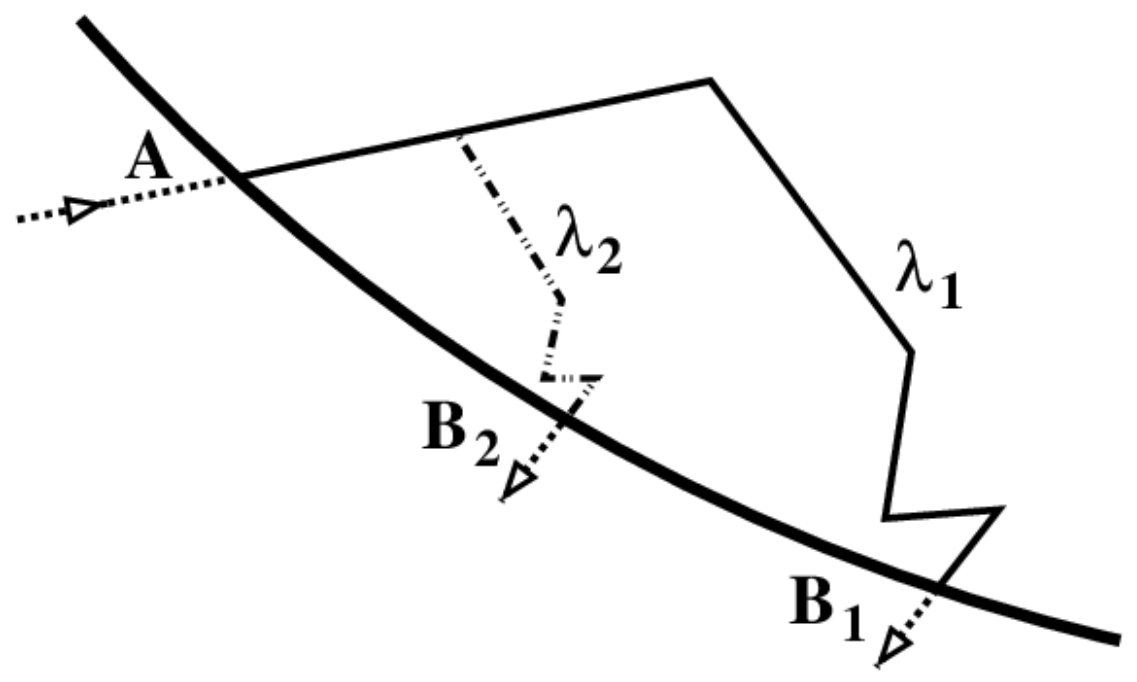

Fig. 3 - Trajectory homothety - Illustration of the quasi-homothety of short trajectories. The second trajectory corresponds to a mean free path half as long as that of the first trajectory $\left(\lambda_{2}=\right.$ $\left.\lambda_{1} / 2\right)$, which for same statistical choices, and neglecting circle curvature, leads to a trajectory length half as long $\left(L_{2} \approx L_{1} / 2\right)$. 